\title{
Telemetry System for Monitoring Data from a Web Server
}

\author{
Wilson G. Salgado, $\mathrm{ENG}^{1}$, Javier I. Hidalgo, $E N G^{2}$, Miguel G. Molina, $\mathrm{MBA}^{1}$ \\ ${ }^{1}$ Escuela Superior Politécnica del Litoral, Ecuador, wsalgado@espol.edu.ec,javihida@espol.edu.ec \\ 2 Escuela Superior Politécnica del Litoral, Ecuador, mgmolina@espol.edu.ec
}

\begin{abstract}
This paper describes the implementation of a telemetry system for detecting, recording and monitoring temperature variation from a remote location in a domestic area. The system consists of a LAN network, including different stages of data detection, conversion and acquisition.

A temperature sensor acquires data for measuring the temperature incidence of a light bul. This data is transmitted through conditioning modules in a LAN network to a receiving station where previously processed data is stored and the time the data was received is shown, to finally display the data in a mobile receiver connected to the $L A N$, showing the data for wireless monitoring.
\end{abstract}

Keywords- Data Base, LAN, Server, Temperature Sensor, Wireless Communication.

\section{INTRODUCTION}

At present, telemetry systems are those that allow us to perform measurements and monitoring of variables remotely, without needing to directly contact the element to be measured [1]. Nowadays this technology has great utility and it is used in several professional fields in industrial and security processes. The system is based on the use of communication networks already structured in a company since some access point (AP) can be used to carry out data transmission [2].

In large and small industries, there are always processes that depend on a temperature factor, where there are limits that the temperature must not exceed or lower, such processes can be the overheating of engines or machinery which could cause great economic losses to the companies in case of a temporary paralysis of the manufacture of the product. Several of these processes are performed 24 hours a day, so a remote monitoring system is of great importance for the good performance of the machines.

Telemetry is the measurement of variables at a distance, without having to be next to a measurement equipment and autonomously, to carry out these measurements strategically placed devices at the measurement points for the sending of the data, the same as are stored without being entered manually.

The design of this system focuses on the transmission of data through a LAN network which consists of a router which will serve as a connection between the temperature sensor and our database. The stored data is displayed by means of an application. For the prototype of this project will be made use of a personal router, which limits our area of coverage, but if the router has internet connection data could be monitored up to a coverage of 30 meters [1].

\section{METHOD OF IMPLEMENTATION}

This article focuses on the design of a prototype of real- time temperature monitoring, from a temperature sensor, a database in the company of a web server, a LAN as a communication medium and an application for Android phones so that the user has easy access to the data.

In the process of data acquisition starts with the storage of data on the server through any device that connects to the same LAN network knowing the IP of the server, continuing with the process the data are acquired from the temperature sensor by means of Arduino and is transmitted with a direct communication to the router, allowing to send the information to the database. Data monitoring consists of sending the information from the database to the cell phone with $\mathrm{Wi}-\mathrm{Fi}$ connection in such a way that the data obtained are displayed.

The experiment consists in recording the temperature, time and ID that identifies the number of the sample, proceeding to regulate the light intensity of a 120 -watt bulb and placing the sensor near the bulb so that the temperature varies and simulates an Industrial environment, allowing us a temperature range from $24^{\circ} \mathrm{C}$ to $99^{\circ} \mathrm{C}$, during the acquisition process the database will be updated manually and at the same time update the data that is monitored in the smart mobile leaving the information with a last value until the When the data is displayed.

\section{A. Reading Data on the LAN.}

The reading of data that will be made in this network will be easy to see for the user because they will have access to it through a cell phone with internet which is now available to all people.

The following block diagram shows the steps of designing data capture and monitoring over a LAN. (See Figure 1).

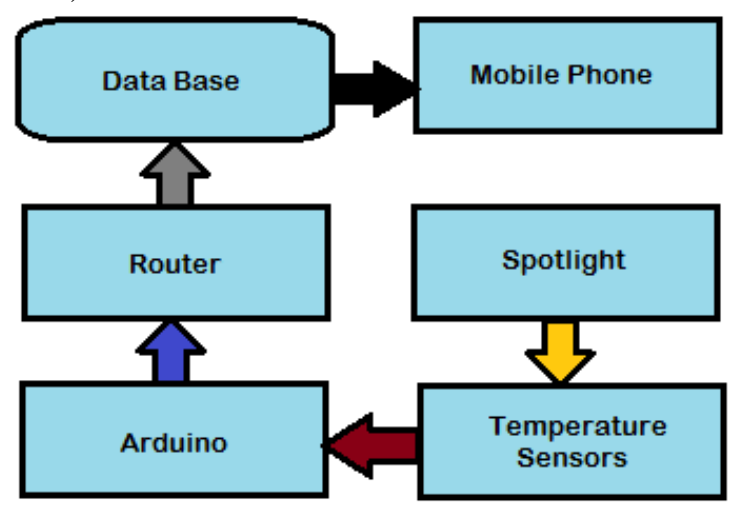

Fig. 1. Block Diagram of the LAN Network.

\section{B. Temperature Measurement.}

For temperature measurement, an analog sensor is used, in this case for simplicity and costs will be used the integrated LM35 that covers temperatures between $-55^{\circ} \mathrm{C}$ 
and $150^{\circ} \mathrm{C}$ with an accuracy of $1^{\circ} \mathrm{C}$.

Because this sensor is a transducer that converts temperature to $\mathrm{mV}$, an analogue to digital conversion should be performed to obtain the actual temperature data in centigrade $\left({ }^{\circ} \mathrm{C}\right)$.

For this A / D conversion, an Arduino UNO will be used. The data to be converted by the Arduino can already be sent by means of an Ethernet shield to the router and thus to arrive at a database.

\section{LAN Server.}

A web server stores HTML documents, images, text files, scripts, and other Web material composed of data (collectively known as content), and distributes this content to customers who request it on the network.

An application server generally manages most (or all) of the business logic and data access functions of the application.

To perform the communication from the host to the server, we use an application for the phone with Android operating system which will be our Host to view the data provided by our server. This application consists of its respective communication software to have access to the database, and in addition a graphic library has been used for a better visualization of the data with respect to the time.

\section{Data Base.}

The database of the communication system is in MySQL, this can be controlled in two ways, through an interface with phpMyAdmin or console [3].

Inside the server we have php files, these files have the function of allowing the requests to arrive at the server and that this can save them in the database or also the function of reading the database.

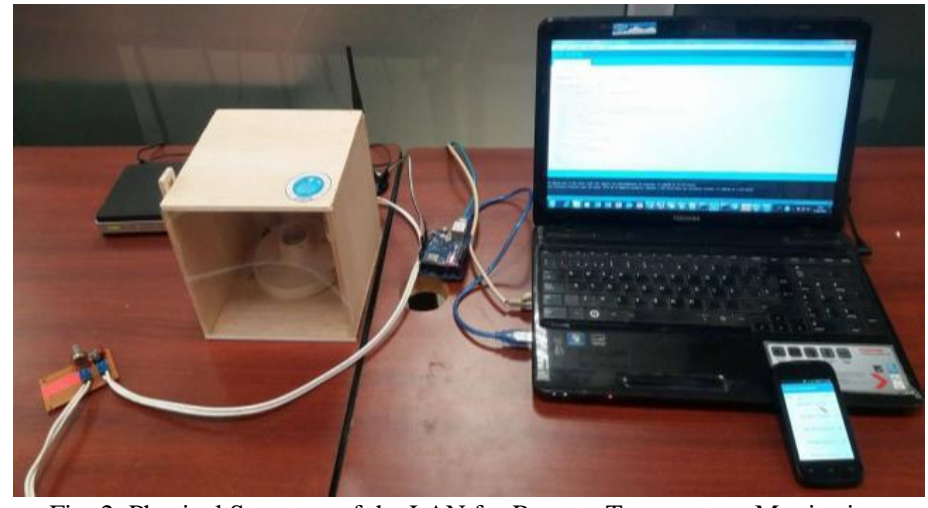

Fig. 2. Physical Structure of the LAN for Remote Temperature Monitoring.

Within the elements of the software we have the Internal Communication Software (SCI), that which exists in the electronic devices and the Software Administrator (SA), has the function of accessing a database, generated from the communication software, performing several processes, such as generating accounts, information or users, data history [5].

Wireless data transmission from the temperature sensor to the cellular device is made possible by standard IEEE 802.11n Wi - Fi technology. Making use of infrastructure, data networks and access points already established, will have cost savings, and will provide an easy solution for the monitoring of machinery and products. This communication system has limitations in the distance, but with the use of the
In addition, for the visualization of the data, the end user has his application in Android, this application allows the connection with the database. This communication is given through a communication software containing the IP address of the server and the address of the files that allow access to the database [4].

Who feeds the database is the temperature sensor LM35 that gives us an analog signal which will be transformed into digital signals by means of an Arduino device, this device has its own IP address and MAC address, which allows us to connect with the Server and can store the information in the database.

\section{E. Elements of Telemetry.}

The system is composed of 3 elements that are, the hardware, software and the means of communication. Among the elements of the hardware are:

1) Temperature sensor LM35: Performs the temperature reading, is a sensor of analog type which transforms the temperature to electricity. Figure 2. The topology is observed.

2) Arduino UNO: Device that will convert the analog temperature signal into digital, in addition will be used to contain the communication software.

3) Ethernet Shield: This device will connect the Arduino to the router.

4) Wireless Router: This device will allow wireless communication to the server that contains the database.

5) PC: it will serve as a server to contain the database. Cell phone: Device that will serve to display the data, graphically. internet it can be accessed from anywhere in the world. Due to the communication standard, will provide easy access to mobile devices and tablets containing the application for access to the database [6].

\section{ANALYSIS OF RESULTS}

In this work, it is proposed to monitor important parameters, integrating various communication technologies, creating a network that describes a communications system with a database, which simplifies and minimizes the time of data collection to do it remotely by accessing the LAN.

An analysis of the temperature data captured, acquired and monitored by the telemetry system was performed, in

$16^{\text {th }}$ LACCEI International Multi-Conference for Engineering, Education, and Technology: "Innovation in Education and Inclusion”, $19-21 \quad$ July $2018, \quad$ Peru. 
which results were obtained that could be represented by means of a graph.

The graph of the data obtained by the sensor in a determined time is observed. The time in which the samples are taken is constant, which allows to capture every event or change in temperature experienced by the focus and through the system and with a stable communication within the coverage area will display the data correctly. (See Figure 3).

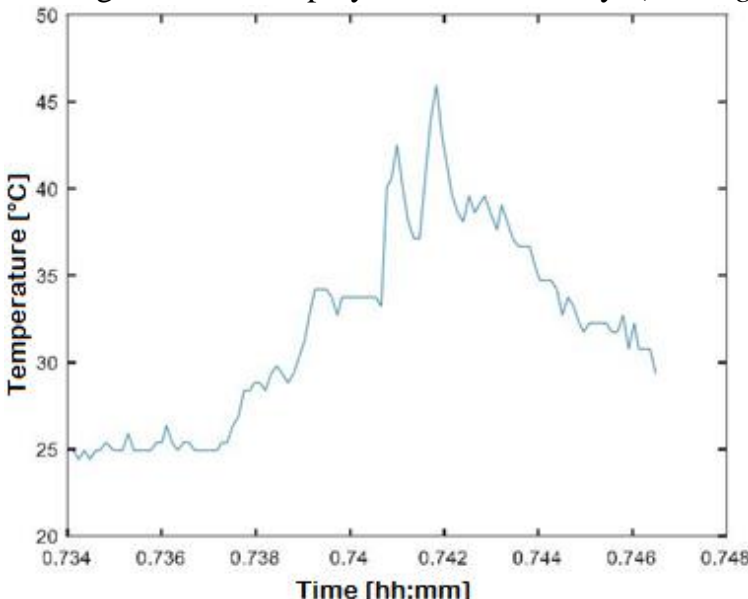

Fig. 3. Graph of the Data Obtained by Means of the Temperature Sensor as a Function of Time.

\section{CONCLUSIONS}

With this project was possible to design and implement a system that allows the transmission of measurements from the regulation of the light intensity of a focus, then obtaining the temperature value by means of an Arduino and can transmit to the web server of Wirelessly to smart mobile wirelessly, using economical and fast access technologies in the middle, such as creating a LAN Network whose infrastructure offers a range of $30 \mathrm{~m}$ coverage.

Per the observations of the sending of data through the Wi - Fi wireless medium it is possible to package the temperature variants of a month, being able to estimate a monitoring and monthly control of the plant.

Per the tests carried out, it was possible to verify that the system designed, transmits the data of the measurements with a minimum of errors, guaranteeing, as far as possible, the delivery of the information through the confirmation of completion, with an average time of a second. The Wi $-\mathrm{Fi}$ technology theory is of $11 \mathrm{Mbit} / \mathrm{s}$ presenting a good quality of service, for this application works properly to the needs of the project.

In addition to the technology used, it was possible to determine that the software programs used as PHP, Arduino Studio and IDE Arduino are very versatile, when establishing communications and data readings from a client and a server using socket. As Java allowed to make connections and execute statements for the database, which are inserted the measurements that arrive through the socket implemented in the PC of the central information station.

All the above allows to conclude satisfactorily that they met the proposed objectives, when designing a system that allows wireless transmission of data read from a temperature sensor to a database, to finally visualize in the application of a smartphone.

$16^{\text {th }}$ LACCEI International Multi-Conference for Engineering, Education, and Technology: "Innovation in Education and Inclusion", $19-21$ July 2018, Lima, Peru.
[1] C. C. L. M. E. Ballesteros, Design of a Wireless Telemetry System to Automate the Reading of Consumption in Water Meters, 2007.

[2] A. S. J. Bobadilla, Communications and Database with JAVA through

[3] A. Gutierres, PHP5 through Examples, Alfaomega, 2011.

[4] H. Forrest, SQL Fundamentals, MacGraw-Hill.

[5] MySQL, Use MySQL with Apache Revision, 2016.

[6] M. Morrison, HTML and XML Designs, MacGraw-Hill, 2011. Examples, 2004. 